\title{
Complex Network Analysis on Fire Risk Evolution of Hazardous Chemical Tanks
}

\author{
Jingjing Li, Xiaoyuan Xu, Xuanya Liu \\ Tianjin Fire Research Institute of MPS, Tianjin 300381, China \\ 危险化学品储罐火灾风险演化复杂网络分析 \\ 李晶晶, 许晓元, 刘咺亚 \\ 公安部天津消防研究所, 天津 300381 , 中国
}

\begin{abstract}
In order to control fire chain risk of hazardous chemical tanks, a method of risk analysis and control on fire evolution of hazardous chemicals is proposed based on the complex networks theory. Firstly, fire risk factors of hazardous chemical tanks were analyzed and screen initial risk events, transitive risk events and terminate risk events. A network model of risk evolution was made based on the logical relationship of risk events. Then the out-in degrees and the clustering coefficients were discussed for risk analysis and the key events of risk evolution were presented. Finally, considering the probability of events occurrence and transformation, the model was converted into a directed network with weight of events. And the shortest paths of risk evolution were calculated by using the Dijkstra algorithm. The risk control measures on disconnecting chains were presented. The results showed that the clustering coefficient of the model was 0.0424, the network aggregation degree was relativelylow.The weights of all the shortest paths were no more than 15. The research can provide the theoretical foundation for the inhibition of risk transfer and control of accident evolution, which has practical significance for guarding the continuous safety operation.
\end{abstract}

key words : fire accidents; risk evolution; complex network; disconnect chains

摘要

为控制危险化学品储罐火灾连锁风险, 基于复杂网络理论提出一种针对储罐火灾 风险演化的分析与控制思路。辨识危险化学 品罐区火灾风险因素, 䇻选初始风险事件、 风险传递事件和末端风险事件, 将风险事件 之间的逻辑关系转化为风险演化网络结构 模型。通过网络节点的出入度分析、聚类系 数分析, 确定风险演化的关键事件。考虑风 险事件的发生和传递概率, 通过赋予边值权 重构建加权有向网络模型, 采用 Dijkstra 算 法求解风险初始事件的演化最短路径, 并提 出断链控制方案。结果表明: 危险化学品储 罐火灾风险演化网络的综合聚类系数 0.0424, 聚集程度偏低; 各初始风险事件的 最短演化路径权重不超过 15。风险演化规律 和最短演化路径研究可为切断事故传递链 条、防止事故升级提供理论依据和借鉴, 对 于保障危险化学品储罐持续安全运行具有 现实意义。

关键词: 火灾事故; 风险演化; 复杂网络; 断链控制

1. 引言

危险化学品罐区常储存易燃、易爆、有 毒物质, 由于人的不安全行为或物的不安全 状态易导致罐内易燃、易爆、有毒物质泄漏, 遇到点火源发生火灾、爆炸等事故, 造成大 
量人员伤亡、财产损失和恶劣社会影响。危 险化学品储罐火灾事故屡见不鲜, 如 2016 年 4 月 22 日江苏德桥仓储有限公司储罐区 交换站发生火灾事故并引燃 2 个汽油储罐, 现场火焰高达二三十米, 直接经济损失 2532.14 万元; 2010 年 7 月 16 日大连中石 油国际储运有限公司原油罐区输油管道因 作业不当发生爆炸, 造成原油大量泄漏并引 发火灾和环境污染, 直接经济损失 22330. 19 万元。因此开展危险化学品储罐区火灾事故 影响因素分析及其演化过程研究对于事故 预防具有现实意义。

目前国内外学者针对危险化学品罐区 火灾事故主要集中在数值模拟 [1-2]、风险 分析 [3-5]、风险预测模型 [6]等方面。风险 分析主要应用事故树、事件树、HAZOP、鱼 骨法、道化学、模糊综合等传统安全分析方 法, 可定性判定火灾风险等级、分析事故发 生的根本因素, 但较少从系统角度分析事故 演化过程中因素逻辑关联与内在机理。复杂 网络模型可为分析因素内在逻辑关系提供 有效的理论支持, 且相关研究已在台风灾害 演化 [7]、电力系统 [8] 风险演化、海底管道 泄漏风险 $[9]$ 防控等方面进行应用。鉴于此, 本文在现有研究基础上, 应用复杂网络模型 对于危险化学品储罐火灾事故风险演化过 程进行分析, 以期为事故预防提供参考。

\section{2. 危险化学品储罐火灾风险演化模型}

\section{1 火灾风险因素分析}

以外浮顶储罐为例, 储罐火灾可分为密 封圈火灾、浮顶溢油火灾、防火堤火灾、浮 筒爆炸、局部或全液面火灾 [9]等类型。储 罐在服役过程中通常会面临自然风险、设备 设施故障风险、人因组织风险、工艺故障风 险、点火源风险等。

1) 自然风险

储罐罐区多为露天设置, 直接经受烈日、 暴雨、严寒的考验。自然条件的严峻性和复 杂性表现为雷击、暴雨、台风等气象能量过 载和地震、地基软弱等自然灾害能量过载。 储罐在雷击天气因防雷系统缺陷容易发生 密封圈起火爆炸事故、暴雨导致浮盘过载下 沉等, 如 1989 年 8 月 12 日黄岛油库发生特
大火灾爆炸事故, 因非金属油罐自身缺陷而 遭受对地雷击、产生感应火花引爆油气。地 质地基条件软弱等可能造成储罐和附属管 道塌陷、移位、沉降, 发生疲劳断裂而发生 泄漏。

2）设备设施风险

储罐设备设施风险包括腐蚀、机械设备 故障、控制系统失效等。涉及易燃、易爆、 剧毒危险化学品的储罐区, 相关规范要求设 置 DCS、紧急切断阀、可燃 (有毒) 气体泄 漏检测报警系统等自动控制设施, 保证危险 源暴露时可及时应急响应, 将事故后果控制 在可接受范围内。2005 年 12 月 11 日, 英国 邦斯菲尔德汽油罐区因仪器仪表失效导致 油料持续向储罐输送, 汽油罐顶溢出。设备 设施机械故障或腐蚀的常见形式有浮盘支 架故障, 罐底或罐壁腐蚀, 管道腐蚀, 与管 道、泵、储罐相连接的阀门、法兰、垫片泄 漏, 罐体或管道焊缝应力腐蚀、材质失效等。

3）人因与组织风险

人因与组织风险包括操作人员责任心 不强、专业技能缺乏和组织管理不完善等, 可能导致罐区内动火等特殊作业安全管理 不严格、人员误操作、日常设施缺乏保养、 事故应急响应不及时等风险因素出现。

4) 工艺故障风险

工艺故障风险表现为工艺生产参数偏 移, 如输送管道油气浓度过高、蒸汽压过高、 油温过高等。

\section{5）点火源风险}

点火源是火灾发生的关键因素。相关研 究成果表明, 雷击、静电、外界火源 (如明 火、烟头、爆炸碎片) 、电火花、金属硫化 物自燃、摩擦与撞击火花等是引发储罐火灾 事故的典型点火源, 其中雷击是最主要的点 火源、静电是引发储罐火灾的常见诱因。

综合上述风险因素分析, 以浮顶罐火灾 事故为例, 将影响危险化学品罐区火灾事故 风险因素划分为 26 个主要风险事件, 如表 1 所示。

\section{2 风险演化网络模型}

根据各风险事件之间的内在逻辑关联, 建立火灾风险演化网络结构模型, 如图 1 所 
表 1 主要风险事件列表

\begin{tabular}{|c|c|c|c|}
\hline 编号 & 事件 & $\begin{array}{l}\text { 编 } \\
\text { 号 }\end{array}$ & 事件 \\
\hline V1 & $\begin{array}{c}\text { 组织管理不 } \\
\text { 善 }\end{array}$ & V2 & $\begin{array}{c}\text { 人因可靠性降 } \\
\text { 低 }\end{array}$ \\
\hline V3 & 人员误操作 & V4 & 维护管理不善 \\
\hline V5 & 设计不合理 & v6 & 生产过程故障 \\
\hline $\mathrm{V} 7$ & $\begin{array}{c}\text { 机械设备故 } \\
\text { 障 }\end{array}$ & V8 & 腐蚀失效 \\
\hline V9 & $\begin{array}{c}\text { 材质瘦劳失 } \\
\text { 效 }\end{array}$ & V10 & 气象能量过载 \\
\hline V11 & $\begin{array}{c}\text { 自然灾害过 } \\
\text { 载 }\end{array}$ & V12 & 管道水击 \\
\hline V13 & 管道破裂 & V14 & 储罐罐体破裂 \\
\hline V15 & $\begin{array}{c}\text { 浮顶沉盘、倾 } \\
\text { 斜 }\end{array}$ & V16 & 密封圈失效 \\
\hline V17 & 储罐泄漏 & V18 & $\begin{array}{c}\text { 储罐可燃蒸汽 } \\
\text { 暴露 }\end{array}$ \\
\hline V19 & 管道泄漏 & V20 & $\begin{array}{c}\text { 洜、阀等附属设 } \\
\text { 施泄漏 }\end{array}$ \\
\hline V21 & 雷击火花 & V22 & 静电火花 \\
\hline V23 & $\begin{array}{c}\text { 电气故障火 } \\
\text { 花 }\end{array}$ & V24 & $\begin{array}{c}\text { 摩擦或撞击火 } \\
\text { 花 }\end{array}$ \\
\hline V25 & 外界明火 & V26 & 储罐火灾 \\
\hline
\end{tabular}

示。该模型为无权有向网络图, 共包含 26 个节点和 55 条边, 分别表示 26 个风险事件 和 55 条演化路径。风险事件中组织管理不 善、气象能量过载、自然灾害过载等节点为 初始风险事件, 储罐火灾为末端风险事件, 其余节点为风险传递事件。由初始风险事件、 风险演化路径、风险传递事件、末端风险事 件共同构成相应的风险演化传递链条。

\section{3. 风险演化复杂网络分析}

\section{1 基于节点出入度的风险分析}

节点度为与该节点相连接的其他节点 数量, 节点 $V i$ 的度数 $k_{i}$ 计算方法为:

$$
k_{i}=\sum_{j} a_{i}
$$

式中: $a_{i}$ 为与节点 $v_{j}$ 之间连接边的数目。

节点作为终点的边数目为节点入度, 表示导 致该风险事件发生的路径个数。节点入度越 大表示风险事件发生的可能性越高、途径越 多、控制难度增加。节点作为起点的边数目 为节点出度, 表示风险事件对后续风险传递 事件的影响程度。节点出度越大表示风险事 件对后续风险演化的综合影响程度越大。节 点出度和入度是基于风险演化网络结构模 型确定。节点出入度分析结果如图 2 所示。

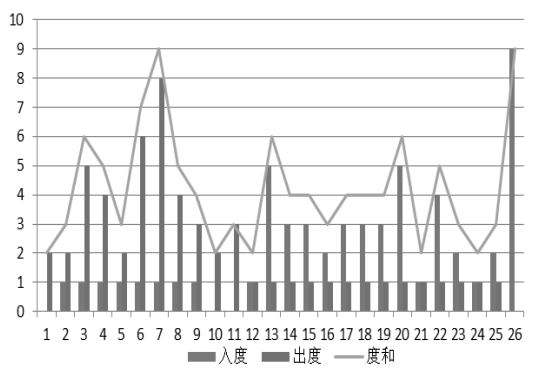

图 2 节点出入度风险分析结果

经计算节点平均度数为 4.23 , 其中机械 设备故障、人因失误、管道破裂、附属设施 泄漏等风险事件度数均超过 6 , 可视为风险 演化网络中关键因素, 表明节点间相互连接 数目较多, 风险事件相互之间联系密切。风 险传递事件中管道破裂、洜阀等附属设施泄 漏、静电火花、储罐罐体破裂、浮盘沉盘或 倾斜、储罐可燃蒸汽暴露等入度相对较大, 导致该风险事件发生的途径较多、风险诱因 比较复杂, 其风险防控难度较高。机械设备 故障、生产过程故障、人员误操作、维护管 理不善、腐蚀失效等风险传递事件的节点出 度较大, 表明风险事件的影响范围较广。度 数较高的风险传递事件在危险化学品储罐 火灾风险防控全生命周期中应重点关注。 


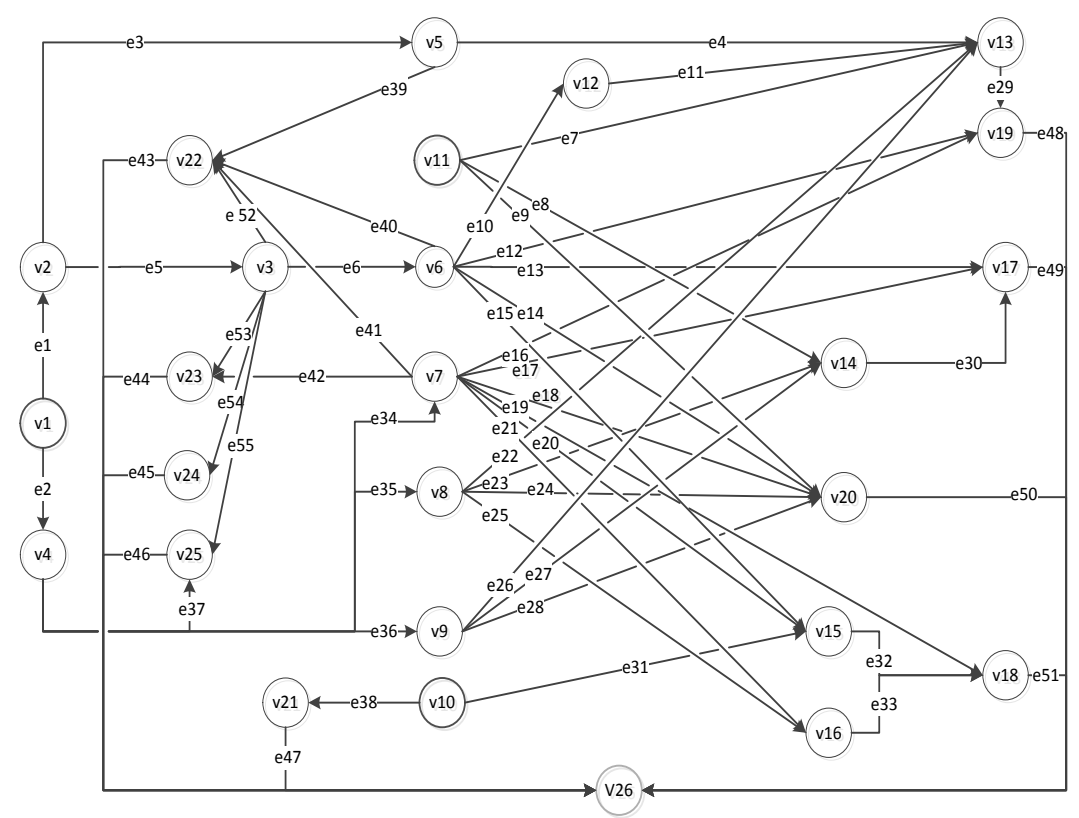

图 1 危险化学品储罐火灾风险演化网络模型

\section{2 基于聚类系数的风险分析}

复杂网络因各节点间相互关联, 常具有 明显的聚类效应。系统结构的聚类系数反应 演化网络节点联系的密切性。节点 Vi 的聚类 系数 Ci 计算方法为:

$$
C_{i}=\frac{2 L_{i}}{n(n-1)}
$$

系统网络整体聚类系数 $\mathrm{C}$ 计算方法为:

$$
\mathrm{C}=\frac{1}{N} \sum_{i=1}^{N} C_{i}
$$

式中: $n$ 为与节点 $V i$ 相连接的节点总 数; $\mathrm{Li}$ 为 $\mathrm{n}$ 个相连接节点实际连接边数目 $; \mathrm{N}$ 为网络的节点总数。

经计算, 该风险演化网络的综合聚类系 数为 0.0424 , 聚集程度偏低。表明风险演化 网络中, 虽然节点和路径较多、事故成因较 为复杂, 但各风险事件之间相互影响并不明 显, 各演化路径存在明显的上下级传递关系。 针对各条演化路径, 制定相适应的断链控制 措施, 可有效达到切断风险路径、降低事故 发生概率的目的。
各节点聚类系数分析结果如图 3 所示。 风险初始事件 V1、V10、V11 的聚类系数均 为 0 , 表明作为风险演化事故链条的初始节 点, 其引发的风险传递事件相互之间相对独 立, 存在多条演化路径交叉的概率较低。风 险传递事件 V16、V18、V22、V7、V6、V3 的 聚类系数非 0 , 表明这些风险传递事件与其 周围相连接的节点之间存在一定的密集联 系, 控制聚类系数较高的风险传递事件可较 为有效抑制风险传递。风险末端事件 V26 的 聚类系数为 0 , 表明风险后果的直接原因事 件相对独立, 不存在较为紧密的联系。

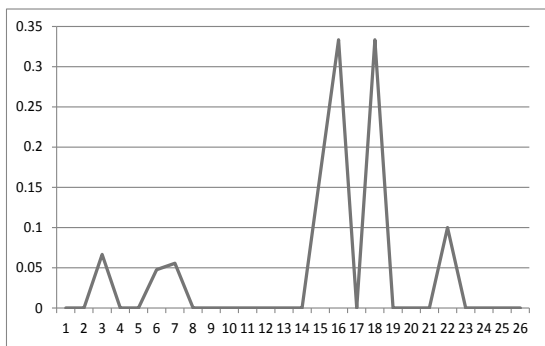

图 3 聚类系数节风险分析结果 


\section{4. 系统风险演化路径分析与断链控制}

\section{1 最短演化路径分析}

在实际运营过程中, 由于各风险因素发 生概率并不完全相同, 可通过权重大小等效 替代发生概率等级, 将复杂网络图转化为加 权有向网络。基于不同演化路径的发生概率, 参照层次分析因素重要度赋值方法, 按照一 定发生、极可能发生、可能发生、不易发生、 不可能发生 5 个层次, 通过专家打分, 分别 对于演化路径赋予 $1 、 3 、 5 、 7 、 9$ 的边权重。 权重值越小表明演化路径发生的概率越高。 采用 Dijkstra 算法进行加权有向网络的最短 演化路径求解。
图 4 中分别用橙色和紫色粗线条突出显 示最短路径。各初始风险事件引发油气泄漏 的最短路径和产生点火源的最短路线如表 2 所示。以雷电恶劣天气为代表的气象能量过 载的初始风险事件其演化路径最短, 其次为 以地震等地质灾害为代表的自然能量过载 作为起点的演化路径, 组织管理不善的演化 路径最长。气象能量过载等因素直接作用于 储罐本体, 故雷电被视为引发浮顶储罐火灾 的主要原因。而组织管理因素属于间接原因, 通过人的不安全行为、物的不安全状态进行 风险传递后再作用于储罐本体, 故其最短演 化路径相对较长。

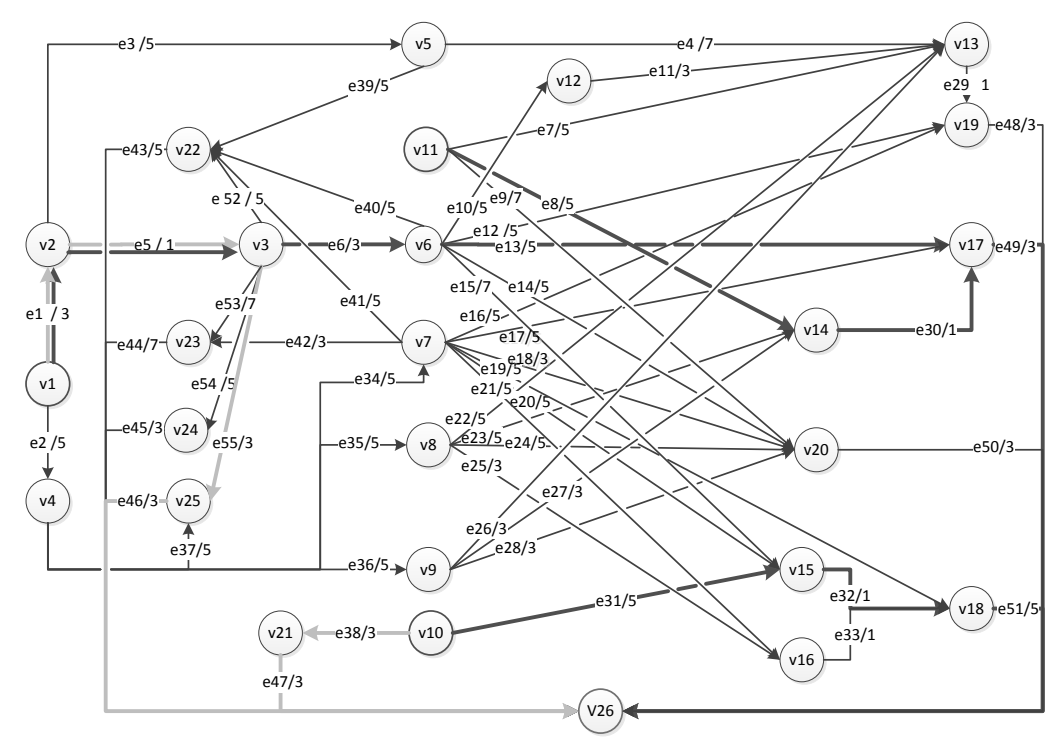

图 4 危险化学品储罐火灾风险演化最短路径

表 2 初始风险事件最短演化路径

\begin{tabular}{c|c|c|c}
\hline 初始风险事件 & 油气泄漏最短路径 D & 权重 w (D) & 权重 w (DD) \\
\hline $\mathrm{V} 1$ & $\mathrm{~V} 1-\mathrm{V} 2-\mathrm{V} 3-\mathrm{V} 6-\mathrm{V} 17-\mathrm{V} 26$ & 15 & 10 \\
\hline $\mathrm{V} 10$ & $\mathrm{~V} 10-\mathrm{V} 15-\mathrm{V} 18-\mathrm{V} 26$ & 11 & 6 \\
\hline $\mathrm{V} 11$ & $\mathrm{~V} 11-\mathrm{V} 14-\mathrm{V} 17-\mathrm{V} 26$ & 9 & 10 \\
\hline
\end{tabular}

\section{2 风险演化路径断链控制}

根据节点度分析结果, 采取控制措施针 对关键因素进行重点防控。从降低火灾风险
发生概率和风险演化的角度来看, 在运营期 间定期开展安全培训教育、提高专业技能和 风险防范意识、完善操作规程和管理制度是 降低人员失误的有效手段; 对于储罐关键设 
备设施开展定期巡查、检测、维护、保养以 提高安全服役周期, 并通过物联网等信息化 手段实现风险特征状态参数的动态监测与 报警, 以及时采取应急响应措施; 管道破裂、 附属设施泄漏等可通过在设计、施工、运营 等全生命周期内采取技术、管理等手段，构 建全生命周期防控体系进行风险早期干预。

根据最短演化路径结果, 提出风险演化 断链方案, 即采取控制措施降低构成最短演 化路径的传递事件发生概率。点火源控制方 面, 提高人员安全意识并加强罐区内动火等 特殊作业安全管理, 禁止罐区出现明火、电 火花等。对于雷电等恶劣天气, 加强罐体雷 电防护本质安全设计、强化各金属附件等电 位连接、消除潜在产生放电的空气间隙, 保 证防雷设施的完好性和可靠性。泄漏源控制 方面, 避免生产过程操作失误导致冒罐等事 故发生。

\section{5. 结论}

（1）构建以风险事件为节点、事件逻 辑关系为边的危险化学品储罐火灾风险演 化网络模型, 应用复杂网络理论通过节点出 入度分析、聚类系数分析、演化路径分析等 可确定导致物质泄漏和形成点火源的关键 因素与最短演化路径。

（2）危险化学品储罐火灾风险演化网 络分析结果表明, 网络模型的综合聚类系数 0.0424 , 聚集程度偏低。各初始风险事件的 最短演化路径权重不超过 15。基于断链思想 针对最短演化路径, 降低风险传递事件出现 概率并切断风险演化路径是防止事故升级 的重要措施。

（3）基于事件概率的风险演化分析, 开展关键因素和风险传递过程的定量研究 是进一步深入研究的重点。

\section{Acknowledgements}

This study was supported by Technology Research Program of MPS (No 2016JSYJD04), TianjinTechnology Research Program (No 16ZXCXSF00080) and Technology Research Program of MPS (No.2016JSYJB32).

\section{致谢}

本研究得到了公安部技术研究计划竞 争性遴选项目 (2016JSYJD04)、安全天津、 科技惠民与可持续发展实验区建设科技专 项 (16ZXCXSF00080)、公安部技术研究计划 （2016JSYJB32）的资助。

\section{参考文献}

[1] 赵金龙, 黄弘, 屈克思, 等. 基于数值模 拟的大型原油储罐热辐射响应研究. 中 南大学学报(自然科学版),48(6):1651-16 58, 2017.

[2] 李庆功, 宋文华, 陈阵,等.基于 FDS 的 大型原油储罐防火堤内池火灾的数值 模拟. 南开大学学报 (自然科学版), 45(1): 77-82, 2012.

[3] Roberto B. Efficient applications of risk analysis in the chemical industry and emergency response. Journal of Risk Analysis and Crisis Response, 1(2):92-101,2011.

[4] 陈福真, 张明广, 王妍, 等. 油气储罐区 多米诺事故耦合效应风险分析. 中国安 全科学学报,27(10):111-116, 2017.

[5] 刘昕亮, 谢飞, 董影超, 等. 液化石油气 站储罐区火灾爆炸危险性分析与安全 措施。中国安全生产科学技术, 7(9):178-182, 2011.

[6] 刘晅亚, 张清林, 秘义行, 等. 大型石油 储罐区火灾风险预测预警技术研究. 消 防科学与技术,31(2):192-196, 2012.

[7] 陈长坤, 纪道溪. 基于复杂网络的台风 灾害演化系统风险分析与控制研究. 灾 害学, 27(1):1-4, 2012.

[8] 夏麟顗, 肖凱金鍂, 刘恩豪. 基于复杂网 络和风险理论的电力系统脆弱性评估. 电子世界, (9):46-47, 2014.

[9] 孟祥坤, 陈国明, 朱红卫. 海底管道泄 漏风险演化复杂网络分析. 中国安全生 产科学技术, 13(4):26-31, 2017.

[10] 任常兴. 基于火灾场景的大型浮顶储罐 区全过程风险防范体系研究. 中国安全 生产科学技术,9(1):68-74, 2014. 